CCNY-HEP-01/06

\title{
Orthogonal basis for the energy eigenfunctions of the Chern-Simons matrix model
}

\author{
Dimitra Karabali ${ }^{a, c}$ and B. Sakita ${ }^{b, c}$ 円 \\ a Department of Physics and Astronomy, Lehman College of the CUNY \\ Bronx, NY 10468 \\ ${ }^{b}$ Physics Department, City College of the CUNY \\ New York, NY 10031 \\ c The Graduate School and University Center, CUNY \\ New York, NY 10016
}

\begin{abstract}
We study the spectrum of the Chern-Simons matrix model and identify an orthogonal set of states. The connection to the spectrum of the Calogero model is discussed.
\end{abstract}

\footnotetext{
${ }^{1}$ e-mail addresses: karabali@alpha.lehman.cuny.edu, sakita@scisun.sci.ccny.cuny.edu
} 


\section{Introduction}

Recently, Susskind [1] proposed a description of quantum Hall effect in terms of a noncommutative $U(1)$ Chern-Simons theory. The fields of this theory are infinite matrices corresponding to an infinite number of electrons confined in the lowest Landau level. Polychronakos, later, proposed [2] a finite matrix model as a regularized version of the noncommutative Chern-Simons theory in order to describe systems of finite many electrons. Although the proposed matrix model seems to reproduce the basic features of the quantum Hall droplets, a precise relation between the matrix model spectrum and the QHE as described by Laughlin wavefunctions is lacking.

A formal mapping between the states of the matrix model and Laughlin states as presented in [3] seems to be non-unitary [4], while coherent state representations of the matrix model states produce wavefunctions with a short distance behavior which does not agree with the Laughlin one [5].

On the other hand the same matrix model was introduced by Polychronakos [6] as being equivalent to the Calogero model [7], a one-dimensional system of particles in an external harmonic osillator potential with mutual inverse-square interactions.

In this paper we analyze the spectrum of the matrix model and present a relatively simple way to identify an orthogonal basis of states. In doing so we make use of known properties of the energy eigenfunctions of the Calogero model. The paper is organized as follows. In section 2 we briefly review the Chern-Simons finite matrix model and its spectrum. In section 3 we analyze the eigenvalue problem and identify an orthogonal basis for the energy eigenstates. The relation to the equivalent eigenvalue problem of the Calogero model is discussed in section 4 and the Appendix.

\section{Chern-Simons matrix model}

The action describing the Chern-Simons matrix model[ is given by [2]

$$
S=\int d t \frac{B}{2} \operatorname{Tr}\left\{\epsilon_{a b}\left(\dot{X}_{a}+i\left[A_{0}, X_{a}\right]\right) X_{b}+2 \theta A_{0}-\omega X_{a}^{2}\right\}+\Psi^{\dagger}\left(i \dot{\Psi}-A_{0} \Psi\right)
$$

where $X_{a}, a=1,2$ are $N \times N$ matrices and $\Psi$ is a complex $N$-vector that transforms in the fundamental of the gauge group $U(N)$,

$$
X_{a} \rightarrow U X_{a} U^{-1}, \quad \Psi \rightarrow U \Psi
$$

The $A_{0}$ equation of motion implies the constraint

$$
G \equiv-i B\left[X_{1}, X_{2}\right]+\Psi \Psi^{\dagger}-B \theta=0
$$

\footnotetext{
${ }^{2}$ For clarification we would like to mention that Smolin has introduced a matrix model, also called matrix Chern-Simons theory [8]. Although there are some common features, the two models are different.
} 
The trace of this equation gives

$$
\Psi^{\dagger} \Psi=N B \theta
$$

Upon quantization the matrix elements of $X_{a}$ and the components of $\Psi$ become operators, obeying the following commutation relations

$$
\begin{aligned}
{\left[\Psi_{i}, \Psi_{j}^{\dagger}\right] } & =\delta_{i j} \\
{\left[\left(X_{1}\right)_{i j},\left(X_{2}\right)_{k l}\right] } & =\frac{i}{B} \delta_{i l} \delta_{j k}
\end{aligned}
$$

The Hamiltonian is

$$
H=\omega\left(\frac{N^{2}}{2}+\sum A_{i j}^{\dagger} A_{j i}\right)
$$

where $A=\sqrt{\frac{B}{2}}\left(X_{1}+i X_{2}\right)$. The system contains $N(N+1)$ oscillators coupled by the constraint (3). As explained in [2], upon quantization, the operator $G$ becomes the generator of unitary rotations of both $X_{a}$ and $\Psi$. The trace part (四) demands that $N B \theta$ being the number operator for $\Psi$ 's is quantized to an integer. The traceless part of the constraint demands the physical states to be singlets of $S U(N)$.

Since the $A_{i j}^{\dagger}$ transform in the adjoint and the $\Psi_{i}^{\dagger}$ transform in the fundamental representation of $S U(N)$, a purely group theoretical argument implies that a physical state being a singlet has to contain $N l \Psi^{\dagger}$ 's, where $l$ is an integer. This leads to the quantization of $B \theta=l$.

Explicit expressions for the states were written down in [3]. The ground state being an $S U(N)$ singlet with the lowest number of $A^{\dagger}$ 's is of the form

$$
\left|\Psi_{g r}\right\rangle=\left[\epsilon^{i_{1} \ldots i_{N}} \Psi_{i_{1}}^{\dagger}\left(\Psi^{\dagger} A^{\dagger}\right)_{i_{2} \ldots}\left(\Psi^{\dagger} A^{\dagger N-1}\right)_{i_{N}}\right]^{l}|0\rangle
$$

where $|0\rangle$ is annihilated by $A$ 's and $\Psi$ 's, while the excited states can be written as

$$
\left|\Psi_{e x c}\right\rangle=\prod_{i=1}^{N}\left(\operatorname{Tr} A^{\dagger i}\right)^{c_{i}}\left[\epsilon^{i_{1} \ldots i_{N}} \Psi_{i_{1}}^{\dagger}\left(\Psi^{\dagger} A^{\dagger}\right)_{i_{2} \ldots(}\left(\Psi^{\dagger} A^{\dagger N-1}\right)_{i_{N}}\right]^{l}|0\rangle
$$

The states (8) have energy $\omega\left(\frac{N^{2}}{2}+l \frac{N(N-1)}{2}+\epsilon\right)$, where $\epsilon=\sum_{i} i c_{i}$. They are degenerate and the degeneracy is given by the number of partitions of $\epsilon$.

The main purpose of this paper is to identify an orthogonal basis for the states (8).

\section{Energy eigenfunctions, orthogonal basis}

As we shall see later, it is convenient to work in the $X$-representation. We define the state $|X, \phi\rangle$ such that

$$
\hat{X}_{1}|X, \phi\rangle=X|X, \phi\rangle \quad \Psi|X, \phi\rangle=\phi|X, \phi\rangle
$$


We normalize the state such that the completeness relation is given by

$$
\int|X, \phi\rangle e^{-\bar{\phi} \phi} d \phi d \bar{\phi} \prod_{i j} d X_{i j}\langle X, \phi|=1
$$

In the $X$-representation the wavefunction corresponding to a particular state of the theory is $\Phi(X, \bar{\phi})=\langle X, \phi|$ state $\rangle$. In particular the wavefunction corresponding to the ground state $(\overline{7})$ is of the form [5]

$$
\Phi_{g r}(X, \bar{\phi})=\left[\epsilon^{i_{1} \ldots i_{N}} \bar{\phi}_{i_{1}}\left(\bar{\phi} A^{\dagger}\right)_{i_{2}} \ldots\left(\bar{\phi} A^{\dagger N-1}\right)_{i_{N}}\right]^{l} e^{-\frac{B}{2} \operatorname{Tr} X^{2}}
$$

where

$$
A_{i j}^{\dagger}=\sqrt{\frac{B}{2}}\left(X_{i j}-\frac{1}{B} \frac{\partial}{\partial X_{j i}}\right)
$$

Since (11) is completely antisymmetric in the $i_{n}$-indices, the differential operator $\frac{\partial}{\partial X_{j i}}$ produces a nonzero contribution only if it acts on the $e^{-\operatorname{Tr} \frac{1}{2} B X^{2}}$ factor. We then have that

$$
\Phi_{g r}(X, \bar{\phi})=(\sqrt{2 B})^{l N(N-1) / 2}\left[\epsilon^{i_{1} \ldots i_{N}} \bar{\phi}_{i_{1}}(\bar{\phi} X)_{i_{2}} \ldots\left(\bar{\phi} X^{N-1}\right)_{i_{N}}\right]^{l} e^{-\frac{B}{2} \operatorname{Tr} X^{2}}
$$

The wavefunction corresponding to the excited state (8) can be written as a linear combination of wavefunctions of the form $(\sqrt{2 B})^{\sum i n_{i}} \prod_{i=1}^{N}\left(\operatorname{Tr} X^{i}\right)^{n_{i}} \Phi_{g r}(X, \bar{\phi})$

Given the constraint $G$, any physical wavefunction has to be a function of $S U(N)$ singlets made out of the hermitian matrix $X$ and the vector $\bar{\phi}$. There are two types of such invariants one can construct 3

$$
\begin{aligned}
S_{n}(\sqrt{2 B} X) & =(\sqrt{2 B})^{n} \operatorname{Tr} X^{n}, \quad n=1, \ldots, N \\
\Xi(\sqrt{2 B} X, \bar{\phi}) & =(\sqrt{2 B})^{N(N-1) / 2} \epsilon^{i_{1} \ldots i_{N}} \bar{\phi}_{i_{1}}(\bar{\phi} X)_{i_{2}} \ldots\left(\bar{\phi} X^{N-1}\right)_{i_{N}}
\end{aligned}
$$

These can be thought of as $N+1$ independent collective variables.

Any physical wavefunction has the general form

$$
\Phi=f\left(S_{n}\right) \Xi^{l} e^{-\frac{B}{2} \operatorname{Tr} X^{2}}
$$

In the $X$-representation the Hamiltonian can be written as

$$
H=\frac{\omega}{2}\left[-\frac{1}{B} \frac{\partial^{2}}{\partial X_{i j} \partial X_{j i}}+B \operatorname{Tr} X^{2}\right]
$$

\footnotetext{
${ }^{3}$ For an $N \times N$ matrix $X$, the Cayley-Hamilton theorem expresses $X^{N}$ as a linear function of $X^{n}$, $\mathrm{n}=1, \ldots, \mathrm{N}-1$ with coefficients which are symmetric functions of the eigenvalues of $X$. Therefore all other invariants which involve $\bar{\phi}$ 's are reduced to $\Xi^{l}$ times a function of $S_{n}$.
} 
We want to solve the eigenvalue problem

$$
H \Phi=E \Phi
$$

where $H$ is given by (16) and $\Phi$ is as in (15). Doing a simple similarity transformation we get

$$
\tilde{H} f\left(S_{n}\right) \Xi^{l}=E f\left(S_{n}\right) \Xi^{l}
$$

where

$$
\begin{aligned}
\tilde{H} & =e^{\frac{B}{2} \operatorname{Tr} X^{2}} H e^{-\frac{B}{2} \operatorname{Tr} X^{2}} \\
& =\omega \sum_{i j} X_{i j} \frac{\partial}{\partial X_{i j}}+\frac{\omega}{2} N^{2}-\frac{\omega}{2 B} \sum_{i j} \frac{\partial^{2}}{\partial X_{i j} \partial X_{j i}}
\end{aligned}
$$

The Hamiltonian $\tilde{H}$ can be written as a sum of two terms

$$
\tilde{H}=\tilde{H}_{0}+\tilde{H}_{-2}
$$

where

$$
\begin{aligned}
\tilde{H}_{0} & =\omega \sum_{i j} X_{i j} \frac{\partial}{\partial X_{i j}}+\frac{\omega}{2} N^{2} \\
\tilde{H}_{-2} & =-\frac{\omega}{2 B} \sum_{i j} \frac{\partial^{2}}{\partial X_{i j} \partial X_{j i}}
\end{aligned}
$$

Since the operator $\tilde{H}_{0}$ essentially counts the number of $X$ 's, one can easily check that

$$
\left[\tilde{H}_{0}, \tilde{H}_{-2}\right]=-2 \tilde{H}_{-2}
$$

In other words

$$
\tilde{H}=e^{\frac{\tilde{H}_{-2}}{2 \omega}} \tilde{H}_{0} e^{-\frac{\tilde{H}_{-2}}{2 \omega}}
$$

This implies that if $P_{k}$ is an eigenfunction of $\tilde{H}_{0}$ then $e^{\tilde{H}_{-2} / 2 \omega} P_{k}$ is an eigenstate of the Hamiltonian $\tilde{H}$. One can easily see that

$$
\begin{aligned}
\tilde{H}_{0} P_{k} & =\left(k+\omega \frac{N^{2}}{2}\right) P_{k} \\
\tilde{H} e^{\frac{\tilde{H}_{-2}}{2 \omega}} P_{k} & =\left(k+\omega \frac{N^{2}}{2}\right) e^{\frac{\tilde{H}_{-2}}{2 \omega}} P_{k}
\end{aligned}
$$

Using (18), (21) we see that in our case $P_{k}$ is of the form

$$
P_{k}=J_{\{\lambda\}}(\sqrt{2 B} X) \Xi^{l}(\sqrt{2 B} X, \bar{\phi})
$$

where $J_{\{\lambda\}}$ is a homogeneous polynomial of the form (the notation will be justified later)

$$
J_{\{\lambda\}}(\sqrt{2 B} X)=\sum_{\left\{n_{i}\right\}} a\left(\left\{n_{i}\right\}\right) \prod_{i}\left(\operatorname{Tr}(\sqrt{2 B} X)^{i}\right)^{n_{i}}
$$


such that $\sum_{i} i n_{i}+l \frac{N(N-1)}{2}=k$.

Going back to the original eigenvalue problem, the energy eigenfunctions are of the form

$$
\Phi=e^{-\frac{B}{2} \operatorname{Tr} X^{2}} e^{\frac{\tilde{H}-2}{2 \omega}}\left(J_{\{\lambda\}} \Xi^{l}\right)
$$

Since

$$
e^{-\frac{B}{2} \operatorname{Tr} X^{2}} e^{\frac{\tilde{H}_{-}}{2 \omega}} X_{i j} e^{-\frac{\tilde{H}_{-2}}{2 \omega}} e^{\frac{B}{2} \operatorname{Tr} X^{2}}=X_{i j}-\frac{1}{B} \frac{\partial}{\partial X_{j i}}
$$

$\Phi$ can be written as

$$
\begin{aligned}
\Phi & =J_{\{\lambda\}}\left[\sqrt{\frac{B}{2}}\left(X_{i j}-\frac{1}{B} \frac{\partial}{\partial X_{j i}}\right)\right] \Xi^{l}\left[\sqrt{\frac{B}{2}}\left(X_{i j}-\frac{1}{B} \frac{\partial}{\partial X_{j i}}\right), \bar{\phi}\right] e^{-\frac{B}{2} \operatorname{Tr} X^{2}} \\
& =J_{\{\lambda\}}\left(A^{\dagger}\right) \Xi^{l}\left(A^{\dagger}, \bar{\phi}\right) e^{-\frac{B}{2} \operatorname{Tr} X^{2}}
\end{aligned}
$$

where $A^{\dagger}$ is given in (12).

There are several basis sets for the polynomials $J_{\{\lambda\}}$. There is a particular one which is orthogonal. This corresponds to choosing $J_{\{\lambda\}}$ 's to be the Jack polynomials [9]-11]. Although, in principle, this can be proven purely within the context of the matrix model itself, an easier proof can be given indirectly by first relating the energy eigenfunctions of the matrix model to the energy eigenfunctions of the Calogero model and then using well known properties of the Calogero eigenfunctions [13]-17]. This is shown in the following section.

\section{Relation to Calogero model}

$X$ being a hermitian matrix, it can be diagonalized by a unitary transformation

$$
X=U x U^{-1} \quad x_{i j}=x_{i} \delta_{i j}
$$

The relation between the matrix model and the Calogero model is achieved by identifying the eigenvalues $x_{i}$ with the one-dimensional particle coordinates of the Calogero system.

One can show that the Laplacian of the matrix model can be written as

$$
\sum_{i j} \frac{\partial}{\partial X_{i j}} \frac{\partial}{\partial X_{j i}}=\frac{1}{\Delta} \sum_{k} \frac{\partial^{2}}{\partial x_{k}^{2}} \Delta-\sum_{k \neq l} \frac{J^{k l} J^{l k}}{\left(x_{k}-x_{l}\right)^{2}}
$$

where $\Delta$ is the Vandermonde determinant defined by

$$
\Delta \equiv \operatorname{det}\left(x_{i}^{N-j}\right)=\epsilon^{k_{1} k_{2} \cdots k_{N}} x_{k_{1}}^{0} x_{k_{2}}^{1} \cdots x_{k_{N}}^{N-1}=\prod_{k<l}\left(x_{k}-x_{l}\right)
$$

and $J^{i j}$ is an operator with the following action on $U$

$$
\left[J^{k l}, U_{i j}\right]=U_{i k} \delta_{l j}, \quad\left[J^{k l}, U_{i j}^{-1}\right]=-U_{l j}^{-1} \delta_{i k}
$$


For completeness we show the detailed derivation of (31) in the appendix.

In writing down the eigenvalue equation for $H$ expressed in terms of the eigenvalues $x_{i}$ and the angular variables $U$, we notice that the term $\sum_{k \neq l} \frac{J^{k l} J^{l k}}{\left(x_{k}-x_{l}\right)^{2}}$ acts only on the $\Xi$ dependence of the wavefunction $\Phi$ in (15). Using the particular parametrization (30), the invariants (14) can be written as

$$
\begin{aligned}
S_{n}(\sqrt{2 B} X) & =(\sqrt{2 B})^{n} \sum_{i=1}^{N} x_{i}^{n} \\
\Xi(\sqrt{2 B} X, \bar{\phi}) & =(\sqrt{2 B})^{N(N-1) / 2} \epsilon^{i_{1} \ldots i_{N}} \bar{\phi}_{i_{1}}(\bar{\phi} X)_{i_{2}}\left(\bar{\phi} X^{2}\right)_{i_{3} \ldots\left(\bar{\phi} X^{N-1}\right)_{i_{N}}} \\
& \left.=(\sqrt{2 B})^{N(N-1) / 2} \epsilon^{i_{1} \ldots i_{N}} \bar{\phi}_{i_{1}}\left(\bar{\phi} U x U^{-1}\right)_{i_{2}}\left(\bar{\phi} U x^{2} U^{-1}\right)_{i_{3} \ldots(\bar{\phi}} U x^{N-1} U^{-1}\right)_{i_{N}} \\
& \left.=(\sqrt{2 B})^{N(N-1) / 2} \operatorname{det}\left(U^{-1}\right) \epsilon^{k_{1} \ldots k_{N}}(\bar{\phi} U)_{k_{1}}(\bar{\phi} U)_{k_{2}} x_{k_{2} \ldots(\bar{\phi}} U\right)_{k_{N}} x_{k_{N}}^{N-1} \\
& =(\sqrt{2 B})^{N(N-1) / 2} \prod_{i<j}\left(x_{i}-x_{j}\right) \prod_{i=1}^{N}(\bar{\phi} U)_{i}
\end{aligned}
$$

This implies

$$
\begin{aligned}
J^{k m} J^{m k} \Xi^{l} & =(\sqrt{2 B})^{l N(N-1) / 2} \Delta^{l} J^{k m} J^{m k} \prod_{i=1}^{N}(\bar{\phi} U)_{i}^{l} \\
& =(\sqrt{2 B})^{l N(N-1) / 2} \Delta^{l} J^{k m} \prod_{i \neq k, m}(\bar{\phi} U)_{i}^{l} l(\bar{\phi} U)_{k}^{l-1}(\bar{\phi} U)_{m}^{l+1} \\
& =l(l+1) \Xi^{l}
\end{aligned}
$$

The Hamiltonian acting on the space of physical wavefunctions (15) can therefore be written as

$$
H=\frac{\omega}{2 B}\left[-\frac{1}{\Delta} \sum_{i} \frac{\partial^{2}}{\partial x_{i}^{2}} \Delta+\sum_{i \neq j} \frac{l(l+1)}{\left(x_{i}-x_{j}\right)^{2}}+B^{2} \sum_{i} x_{i}^{2}\right]
$$

The expression $\Delta H \Delta^{-1}$ coincides with the Hamiltonian of the Calogero model. This actually implies that $\Delta e^{-\frac{B}{2} \operatorname{Tr} X^{2}} e^{\frac{\tilde{H}_{-2}}{2 \omega}} J_{\{\lambda\}}(\sqrt{2 B} X) \Xi^{l}(\sqrt{2 B} X, \bar{\phi})$ are energy eigenfunctions of the Calogero model.

Using (31), (34), (35) one can show that

$$
\Delta e^{-\frac{B}{2} \operatorname{Tr} X^{2}} e^{\frac{\tilde{H}_{-2}}{2 \omega}} J_{\{\lambda\}}(\sqrt{2 B} X) \Xi^{l}(\sqrt{2 B} X, \phi) \sim \Delta^{l+1} e^{-\frac{B}{2} \operatorname{Tr} X^{2}} e^{-\frac{\hat{O}_{L}}{4 B}} J_{\{\lambda\}}\left(\sqrt{2 B} x_{i}\right)
$$

where

$$
\hat{O}_{L}=\sum_{i} \frac{\partial^{2}}{\partial x_{i}^{2}}+(l+1) \sum_{i \neq j} \frac{1}{x_{i}-x_{j}}\left(\frac{\partial}{\partial x_{i}}-\frac{\partial}{\partial x_{j}}\right)
$$

Comparing (37) to the orthogonal basis of the energy eigenfunctions of the Calogero model [12]-[17, we conclude that the polynomials $J_{\{\lambda\}}$ ought to be the symmetric Jack 
polynomials. The inhomogeneous polynomials $e^{-\frac{\hat{O}_{L}}{4 B}} J_{\{\lambda\}}\left(\sqrt{2 B} x_{i}\right)$ are the symmetric Hi-Jack polynomials [14 - 17] which provide an orthogonal basis for the Calogero model with the integration measure [15]-16]

$$
\Delta^{2 l+2} e^{-B \sum x_{i}^{2}} \prod_{i} d x_{i}
$$

The symmetric Jack polynomials $J_{\{\lambda\}}$ of degree $\lambda$ are defined [9]-11] in terms of the monomial functions $\prod_{i} x_{i}^{\lambda_{i}}$, where $\lambda=\sum_{i} \lambda_{i}$. Let $\{\lambda\}$ indicate the partitions $\left\{\lambda_{1}, \ldots, \lambda_{N}\right\}$. To a partition $\{\lambda\}$ we associate the symmetric monomial function $m_{\{\lambda\}}=\sum_{P} \prod_{i} x_{i}^{\lambda_{i}}$, where the sum is over all distinct permutations $P$. For example $m_{\{2,1\}}=\sum_{i, j} x_{i}^{2} x_{j}$ for $i \neq j$. $m_{\{\lambda\}}\left(x_{1}, x_{2}, \ldots, x_{N}\right)=0$ if $N$ is smaller than the number of parts of the partition $\{\lambda\}$. The symmetric Jack polynomials have the following expansion in terms of $m_{\{\lambda\}}$ 's:

$$
J_{\{\lambda\}}\left(x_{i}\right)=m_{\{\lambda\}}\left(x_{i}\right)+\sum_{\{\mu\}<\{\lambda\}} v_{\{\mu \lambda\}} m_{\{\mu\}}\left(x_{i}\right)
$$

$v_{\{\mu \lambda\}}$ are some coefficients which depend on $l$ and $\{\mu\}<\{\lambda\}$ defines a partial ordering such that

$$
\{\mu\}<\{\lambda\} \leftrightarrow \mu=\lambda \text { and } \sum_{i=1}^{j} \mu_{i}<\sum_{i=1}^{j} \lambda_{i} \text { for all } j=1,2, \cdots, N
$$

Since there is no generic formula, we write down the first few symmetric Jack polynomials, relevant for the construction of the first, second and third excited states.

$$
\begin{aligned}
& J_{\{0\}}=1 \\
& J_{\{1\}}=m_{\{1\}} \\
& J_{\{2\}}=m_{\{2\}}+\frac{2(l+1)}{l+2} m_{\{1,1\}} \\
& J_{\{1,1, \cdots, 1\}}=m_{\{1,1, \cdots, 1\}} \\
& J_{\{2,1\}}=m_{\{2,1\}}+\frac{6(l+1)}{2 l+3} m_{\{1,1,1\}} \\
& J_{\{3\}}=m_{\{3\}}+\frac{3(l+1)}{l+3} m_{\{2,1\}}+\frac{6(l+1)^{2}}{(l+2)(l+3)} m_{\{1,1,1\}}
\end{aligned}
$$

Using these one can explicitly check that the corresponding Hi-Jack polynomials $e^{-\frac{\hat{O}_{L}}{4 B}} J_{\{\lambda\}}$ are orthogonal with the integration measure (39).

Since $J_{\{\lambda\}}$ 's are symmetric, they can also be expressed in terms of $\prod_{i}\left(S_{i}\right)^{n_{i}}=\prod_{i}\left(\operatorname{Tr} X^{i}\right)^{n_{i}}$ where $\sum_{i} i n_{i}=\lambda$. It is this dependence which is implied in $J_{\{\lambda\}}(\sqrt{2 B} X)$ in $(26)$ and in $J_{\{\lambda\}}\left(A^{\dagger}\right)$ in $(29)$.

Going back to the matrix model eigenfunctions (29) and recalling that $\prod_{i j} d\left[X_{i j}\right]=$ $\Delta^{2}[d U] \prod_{i} d x_{i}$, we conclude that the states

$$
\Phi_{\{\lambda\}}=J_{\{\lambda\}}\left(A^{\dagger}\right) \Xi^{l}\left(A^{\dagger}, \phi\right) e^{-\frac{B}{2} \operatorname{Tr} X^{2}}
$$


where $J_{\{\lambda\}}$ 's are the symmetric Jack polynomials, provide an orthogonal basis for the matrix energy eigenfunctions

$$
\int \Phi_{\{\lambda\}}^{*} \Phi_{\left\{\lambda^{\prime}\right\}} \prod_{i j} d X_{i j} d \phi d \bar{\phi} e^{-\phi \bar{\phi}}=0 \quad \text { for } \quad\{\lambda\} \neq\left\{\lambda^{\prime}\right\}
$$

Using now eq.(10), we can write an orthogonality relation for the states of the matrix model independent of representation, namely

$$
\left\langle\Psi_{\{\lambda\}} \mid \Psi_{\left\{\lambda^{\prime}\right\}}\right\rangle=0 \quad \text { for } \quad\{\lambda\} \neq\left\{\lambda^{\prime}\right\}
$$

where $\left|\Psi_{\{\lambda\}}\right\rangle=J_{\{\lambda\}}\left(A^{\dagger}\right)\left[\epsilon^{i_{1} \ldots i_{N}} \Psi_{i_{1}}^{\dagger}\left(\Psi^{\dagger} A^{\dagger}\right)_{i_{2} \ldots}\left(\Psi^{\dagger} A^{\dagger N-1}\right)_{i_{N}}\right]^{l}|0\rangle$

The use of the $X$-representation and the resulting connection to the Calogero model was very helpful in identifying the Jack polynomial dependence of an orthogonal basis for the excited states, but the final result (45) is independent of representation.

\section{Acknowledgements}

We would like to thank A.P. Polychronakos for useful discussions. We also thank S. Meljanac and L. Jonke for pointing out a minor error in the original version. This work was supported in part by the NSF grant PHY-9970724 and a PSC-32 CUNY award.

\section{Appendix}

Using the "polar" decomposition (30) for X we find

$$
\begin{aligned}
d X & =U\left(d x+\left[U^{-1} d U, x\right]\right) U^{-1} \\
d X_{i j} & =U_{i k} U_{k j}^{-1} d x_{k}-U_{i k} U_{l j}^{-1}\left(x_{k}-x_{l}\right)\left(U^{-1} d U\right)_{k l}
\end{aligned}
$$

Using the parametrization $U=e^{i \sum_{\alpha} t_{\alpha} \theta_{\alpha}}$ we get

$$
\left(U^{-1} d U\right)_{k l}=\left(U^{-1} \frac{\partial U}{\partial \theta_{\alpha}}\right)_{k l} d \theta_{\alpha} \equiv e_{k l}^{\alpha}(\theta) d \theta_{\alpha}
$$

Thus

$$
d x_{k}=\left(U^{-1} d X U\right)_{k k} \quad ; \quad\left(U^{-1} d U\right)_{k l}=-\frac{\left(U^{-1} d X U\right)_{k l}}{x_{k}-x_{l}} \text { for } k \neq l
$$

Using this we find

$$
\frac{\partial}{\partial X_{i j}}=\sum_{k} U_{k i}^{-1} U_{j k} \frac{\partial}{\partial x_{k}}-\sum_{k \neq l} \frac{U_{k i}^{-1} U_{j l}}{\left(x_{k}-x_{l}\right)} J^{k l}
$$

where

$$
J^{k l}=\sum_{\alpha} e_{\alpha}^{k l} \frac{\partial}{\partial \theta_{\alpha}}
$$


and $e_{\alpha}^{k l}$ is the inverse of $e_{k l}^{\alpha}$, such that

$$
\begin{aligned}
& \sum_{k l} e_{\alpha}^{k l}(\theta) e_{k l}^{\beta}(\theta)=\delta_{\alpha \beta} \\
& \sum_{p} e_{\alpha}^{k l}(\theta) e_{k^{\prime} l^{\prime}}^{\alpha}(\theta)=\delta_{k k^{\prime}} \delta_{l l^{\prime}}
\end{aligned}
$$

Expression (50) implies that the action of $J^{k l}$ on $U$ is as follows

$$
\left[J^{k l}, U_{i j}\right]=U_{i k} \delta_{l j}
$$

Further

$$
\left[J^{k l}, J^{m n}\right]=J^{k n} \delta_{l m}-J^{m l} \delta_{k n}
$$

The matrix Laplacian can now be rewritten as

$$
\begin{aligned}
\sum_{i j} \frac{\partial}{\partial X_{i j}} \frac{\partial}{\partial X_{j i}} & =\sum_{i j}\left[\sum_{k} U_{k i}^{-1} U_{j k} \frac{\partial}{\partial x_{k}} \sum_{k^{\prime}} U_{k^{\prime} j}^{-1} U_{i k^{\prime}} \frac{\partial}{\partial x_{k}^{\prime}}\right. \\
& -\sum_{k} U_{k i}^{-1} U_{j k} \frac{\partial}{\partial x_{k}} \sum_{k^{\prime} \neq l^{\prime}} \frac{U_{k^{\prime} j}^{-1} U_{i l^{\prime}}}{\left(x_{k}^{\prime}-x_{l}^{\prime}\right)} J^{k^{\prime} l^{\prime}} \\
& -\sum_{k \neq l} \frac{U_{k i}^{-1} U_{j l}}{\left(x_{k}-x_{l}\right)} J^{k l} \sum_{k^{\prime}} U_{k^{\prime} j}^{-1} U_{i k^{\prime}} \frac{\partial}{\partial x_{k}^{\prime}} \\
& +\sum_{k \neq l} \frac{U_{k i}^{-1} U_{j l}}{\left(x_{k}-x_{l}\right)} J^{k l} \sum_{k^{\prime} \neq l^{\prime}} \frac{U_{k^{\prime} j}^{-1} U_{i l^{\prime}}}{\left(x_{k}^{\prime}-x_{l}^{\prime}\right)} J^{\left.k^{\prime} l^{\prime}\right]}
\end{aligned}
$$

The first term is $\sum_{k} \frac{\partial^{2}}{\partial x_{k}^{2}}$, the second term is zero, the third is $\sum_{k \neq l} \frac{1}{\left(x_{k}-x_{l}\right)}\left(\frac{\partial}{\partial x_{k}}-\frac{\partial}{\partial x_{l}}\right)$, and the last is $-\sum_{k \neq l} \frac{J^{k l} J^{k}}{\left(x_{k}-x_{l}\right)^{2}}$. Thus we obtain

$$
\sum_{i j} \frac{\partial}{\partial X_{i j}} \frac{\partial}{\partial X_{j i}}=\frac{1}{\Delta} \sum_{k} \frac{\partial^{2}}{\partial x_{k}^{2}} \Delta-\sum_{k \neq l} \frac{J^{k l} J^{l k}}{\left(x_{k}-x_{l}\right)^{2}}
$$

where we used

$$
\sum_{k} \frac{\partial^{2}}{\partial x_{k}^{2}}+\sum_{k \neq l} \frac{1}{\left(x_{k}-x_{l}\right)}\left(\frac{\partial}{\partial x_{k}}-\frac{\partial}{\partial x_{l}}\right)=\frac{1}{\Delta} \sum_{k} \frac{\partial^{2}}{\partial x_{k}^{2}} \Delta
$$

and $\Delta=\prod_{k<l}\left(x_{k}-x_{l}\right)$.

\section{References}

[1] L. Susskind, hep-th/0101029.

[2] A.P. Polychronakos, JHEP 0104 (2001) 011, hep-th/0103013. 
[3] S. Hellerman and M. Van Raamsdonk, hep-th/0103179.

[4] A.P. Polychronakos, hep-th/0106011.

[5] D. Karabali and B. Sakita, hep-th/0106016.

[6] A.P. Polychronakos, Phys. Lett. B266 (1991) 29.

[7] F. Calogero, J. Math. Phys. 12 (1971) 419.

[8] L. Smolin, Phys. Rev. D57 (1998) 6216; Nucl. Phys. B591 (2000) 227; hep-th/0006137.

[9] H. Jack, Proc. R. Soc. Edinburg (A) 69 (1970) 1.

[10] R.P. Stanley, Adv. Math. 77 (1988) 76.

[11] I.G. Macdonald, Symmetric Functions and Hall Polynomials, 2nd edition, Oxford, Clarendon press, 1995.

[12] H. Ujino and M. Wadati, J. Phys. Soc. Japan 64 (1995) 2703; 65 (1996) 653; 65 (1996) 2423; 66 (1997) 345; T.H. Baker and P.J. Forrester Commun. Math. Phys. 188 (1997) 175; J.F. van Diejen, Commun. Math. Phys. 188 (1997) 467.

[13] M. Lassalle, C.R. Acad. Sci. Paris, t. Series I 312 (1991) 725; 313 (1991) 579.

[14] K. Sogo, J. Phys. Soc. Japan 65 (1996) 3097.

[15] T.H. Baker and P.J. Forrester, Nucl. Phys. B492 (1997) 682.

[16] A. Nishino, H. Ujino and M. Wadati, cond-mat/9803284.

[17] N. Gurappa and P.K. Panigrahi, Phys. Rev. B59 (1999) R2490; Phys. Rev. B62 (2000) 1943. 